\title{
Pollution Physique Et Bactériologique De L'eau Du Fleuve Dans La Basse Vallée De L'ouémé Pendant Les Périodes De Basses Et Hautes Eaux Au Bénin
}

\begin{abstract}
Adjagodo A.
Institut National de l'Eau (INE), Université d'Abomey-Calavi (UAC), Cotonou, Bénin.

Laboratoire des Normes et Contrôle de Qualité Microbiologique, Nutritionnelle et Pharmacologique (LNCQ ${ }^{\mathrm{MNP}}$ ), Faculté des Sciences et Techniques (FAST), Université d'Abomey-Calavi (UAC), Cotonou, Bénin. Chaire Internationale de Physique Mathématique et Applications (CIPMA-

Chaire UNESCO), Université d'Abomey-Calavi, Cotonou.
\end{abstract}

\section{Agassounon Djikpo Tchibozo M.}

Laboratoire des Normes et Contrôle de Qualité Microbiologique,

Nutritionnelle et Pharmacologique (LNCQ $\left.{ }^{\mathrm{MNP}}\right)$, Faculté des Sciences et Techniques (FAST), Université d'Abomey-Calavi (UAC), Cotonou, Bénin.

\section{Kelomè N.C.}

Laboratoire de Géologie, Mines et Environnement, Faculté des Sciences et Techniques (FAST), Université d'Abomey-Calavi (UAC), Cotonou, Bénin.

\section{Vissin E.W.}

Laboratoire Pierre Pagney "Climats, Eau, Ecosystèmes et Développement »,

Faculté des Lettres, Arts et Sciences Humaines, Université d'AbomeyCalavi, Cotonou, Bénin.

\section{Agbossou E.}

Institut National de l'Eau (INE), Université d'Abomey-Calavi (UAC), Cotonou, Bénin.

Laboratoire d'Hydraulique et de Maîtrise de l'Eau (LHME), Université d'Abomey-Calavi (UAC), Cotonou, Bénin.

Doi: 10.19044/esj.2017.v13n33p167 URL:http://dx.doi.org/10.19044/esj.2017.v13n33p167

Abstract

The objective of this study is to assess the water river quality of the Lower Valley of Ouémé in Benin. Two river water sampling campaigns were carried out during low and high water. At each sampling campaign, eleven (11) sampling sites were selected and at each site four samples were taken, making a total of 44 water samples. Hydrogen potential, dissolved oxygen, suspended matter and turbidity were measured in situ. Fecal contamination germs such as total coliforms, thermotolerant coliforms, Escherichia coli and 
fecal enterococci were counted by the membrane filtration technique. The results of the physical analyzes reveal that the $\mathrm{pH}$ measured in the water samples conformed to the WHO standard except turbidity. The results of the bacteriological analyzes in $100 \mathrm{~mL}$ of water sample of the river reveal average bacterial loads in periods of low and high waters which vary respectively from $2.57 .10^{2} \pm 451 \mathrm{CFU}$ and $3.35 .10^{2} \pm 459 \mathrm{CFU}$ for total coliforms; $1.42 .10^{2} \pm 315 \mathrm{CFU}$ and $1.55 .10^{2} \pm 251$ for thermotolerant coliforms; $0,79.10^{2} \pm 216 \mathrm{CFU}$ and $1.55 .10^{2} \pm 251$ for E. coli. The Enterococci are absent in the waters during these two periods. The high burden of these germs counted in the high water period may be due runoff phenomenon and unhygienic behavior observable in the study area.

Keywords: Water resources, Faecal contamination, Physical quality, Unhygienic behavior

\section{Résume}

L'objectif de l'étude est d'évaluer la qualité de l'eau du fleuve de la Basse Vallée de l'Ouémé au Bénin. Deux campagnes d'échantillonnage d'eau du fleuve ont été effectuées en période de basses et hautes eaux. A chaque campagne d'échantillonnage, onze (11) sites de prélèvement ont été retenus et sur chaque site quatre échantillons ont été prélevés soit un total de 44 échantillons d'eau. Le potentiel hydrogène, l'oxygène dissous, les matières en suspension et la turbidité ont été mesurés in situ. Les germes de contamination fécale tels que Coliformes totaux, Coliformes thermotolérants, Escherichia coli et Entérocoques fécaux ont été dénombrés par la technique de filtration sur membrane. Les résultats des analyses physiques révèlent que le $\mathrm{pH}$ mesuré dans les échantillons d'eau est conforme à la norme de l'OMS excepté la turbidité. Les résultats des analyses bactériologiques dans $100 \mathrm{~mL}$ d'échantillon d'eau du fleuve révèlent des charges bactériennes moyennes en périodes de basses et hautes eaux qui varient respectivement de $2,57 \cdot 10^{2} \pm$ 451 UFC et $3,35.10^{2} \pm 459$ UFC pour Coliformes totaux ; $1,42.10^{2} \pm 315$ UFC et $1,55.10^{2} \pm 251$ pour Coliformes thermotolérants ; 7,9.10 ${ }^{1} \pm 216$ UFC et $1,55.10^{2} \pm 251$ pour E. coli. Les Entérocoques sont absents dans les eaux pendant ces deux périodes. La forte charge en ces germes dénombrés au cours de la période de hautes eaux peut être due au phénomène de ruissellement et à des comportements non hygiéniques observables dans la zone d'étude.

Mots-clés : Ressource en eau, Contamination fécale, Qualité physique, Comportements non hygiéniques 


\section{Introduction}

Les eaux de surface sont exploitées à des fins diverses. D'après AfriMehennaoui et al. (2009), elles constituent la source la plus menacée par les activités humaines. Elles sont les plus exposées à des pollutions, car ces eaux servent de dépotoirs des déchets divers (déchets domestiques, agricoles et pastoraux) et sont des collecteurs d'eaux usées provenant des agglomérations (Bagalwa, 2013). Selon Saab et al. (2007), les eaux de surface sont le lieu de déversements abusifs d'effluents industriels et domestiques sans traitement préalable. Ces eaux sont aussi le lieu d'aisance de la population n'ayant pas accès à un système de défécation approprié. C'est le cas du fleuve de la Basse Vallée de l'Ouémé. Les populations riveraines de la Basse Vallée de l'Ouémé défèquent directement dans l'eau du fleuve à travers des latrines publiques installées sur ce fleuve ou parfois dans les brousses. Or, cette eau est utilisée par la population pour des activités domestiques, voire la boisson, sans être traitée ni contrôlée (Adjagodo et al., 2016). La consommation de cette eau sans traitement peut causer des maladies hydriques. Alors, l'eau vitale pour l'existence, peut être aussi une source de maladie hydrique (Ndahama et al., 2014). D'après l'Organisation Mondiale de la Santé (OMS), 361000 enfants de moins de 5 ans meurent chaque année de maladies diarrhéiques à cause d'un accès insuffisant à l'eau potable et aux moyens d'assainissement et d'hygiène (WHO, 2017). Il faut aussi noter que la consommation d'eau douce engendre inéluctablement une pollution par le rejet des eaux usées menaçant directement la qualité de l'eau et l'intégrité des écosystèmes aquatiques voire la santé humaine (Errochdi et $a l ., 2012$ ). La qualité de l'eau est alors un facteur influençant l'état de santé et de mortalité à la fois chez l'homme et les animaux (Kazi et al., 2009). Lorsque le milieu aquatique reçoit des rejets d'origine animale ou anthropique, le nombre et le type de bactéries présents sont capables de rendre l'eau impropre à l'utilisation humaine (Hébert et Légaré, 2000). La qualité d'une telle eau est ainsi menacée. Cette qualité évolue avec les saisons et d'une région à l'autre, même en absence de pollution (Aguiza et al., 2014). Alors, cette étude a pour objectif d'évaluer la qualité physique et bactériologique de l'eau du fleuve de la Basse Vallée de l'Ouémé en fonction des saisons de l'année.

\section{Matériel et méthodes}

\section{Localisation de la zone d'étude}

La Basse Vallée de l'Ouémé (figure 1) est située entre $6^{\circ} 25^{\prime}$ et $6^{\circ} 57^{\prime}$ de Latitude Nord et $2^{\circ} 21^{\prime}$ 'et $2^{\circ} 38^{\prime}$ de Longitude Est au Sud du Bénin dans le Département de l'Ouémé. 


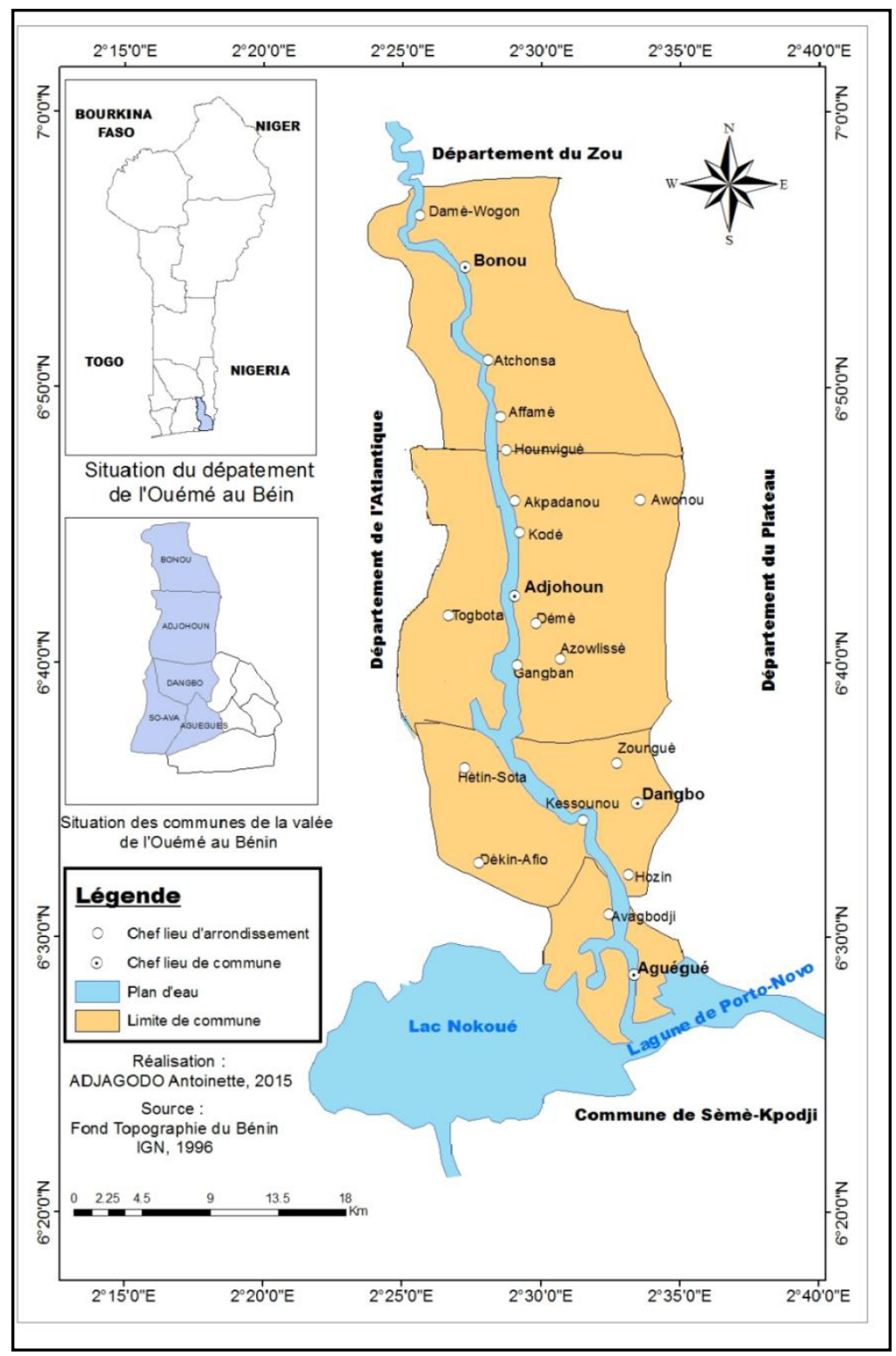

Figure 1 : Carte de localisation de la zone d'étude

La Basse Vallée de l'Ouémé est alimentée par le plus grand fleuve du Bénin qu'est le fleuve Ouémé $(510 \mathrm{~km})$. Ce fleuve traverse quatre (04) Communes de la Basse Vallée de l'Ouémé à savoir Dangbo, Aguégués, Adjohoun et Bonou. Le climat est de type subéquatorial à quatre saisons d'inégale répartition, deux saisons pluvieuses et deux saisons sèches. Le régime hydrologique de 1 'Ouémé est caractérisé par un débit minimal au mois de mars et un débit maximal pendant la période de hautes eaux au mois 
de septembre, mais la période de basses eaux s'étend de janvier à mai inclus. La crue arrive en juin et le débit croît jusqu'en septembre ; il se maintient au voisinage du maximum pendant le mois d'octobre (Moniod, 1973).

\section{Echantillonnage}

Les points d'eau échantillonnés (figure 2) ont été choisis verticalement au niveau du fleuve Ouémé en tenant compte de la distance des activités anthropogéniques polluantes (s'exerçant de $0,50 \mathrm{~m}$ à $1000 \mathrm{~m} \mathrm{du}$ fleuve) et aussi des fortes sollicitations de l'eau du fleuve par la population. Onze (11) sites de prélèvement ont été retenus et sur chaque site quatre (04) échantillons ont été prélevés soit 44 échantillons par période. Les eaux ont été échantillonnées pendant la période de basses eaux (avril 2016) et de hautes eaux (septembre 2016) pour les analyses bactériologiques. Les échantillons d'eau ont été prélevés à $0,50 \mathrm{~m}$ de la surface dans des bouteilles en verre de $500 \mathrm{~mL}$ stérilisées. Chaque échantillon a été identifié au moyen d'une fiche de prélèvement (date, lieu, heure du prélèvement, numéro et mesures in situ). Les échantillons d'eau prélevés ont été ensuite transportés au laboratoire sous un régime de froid. Les coordonnées des points chantillonnés ont été prises avec le GPS de type GARMIN. 


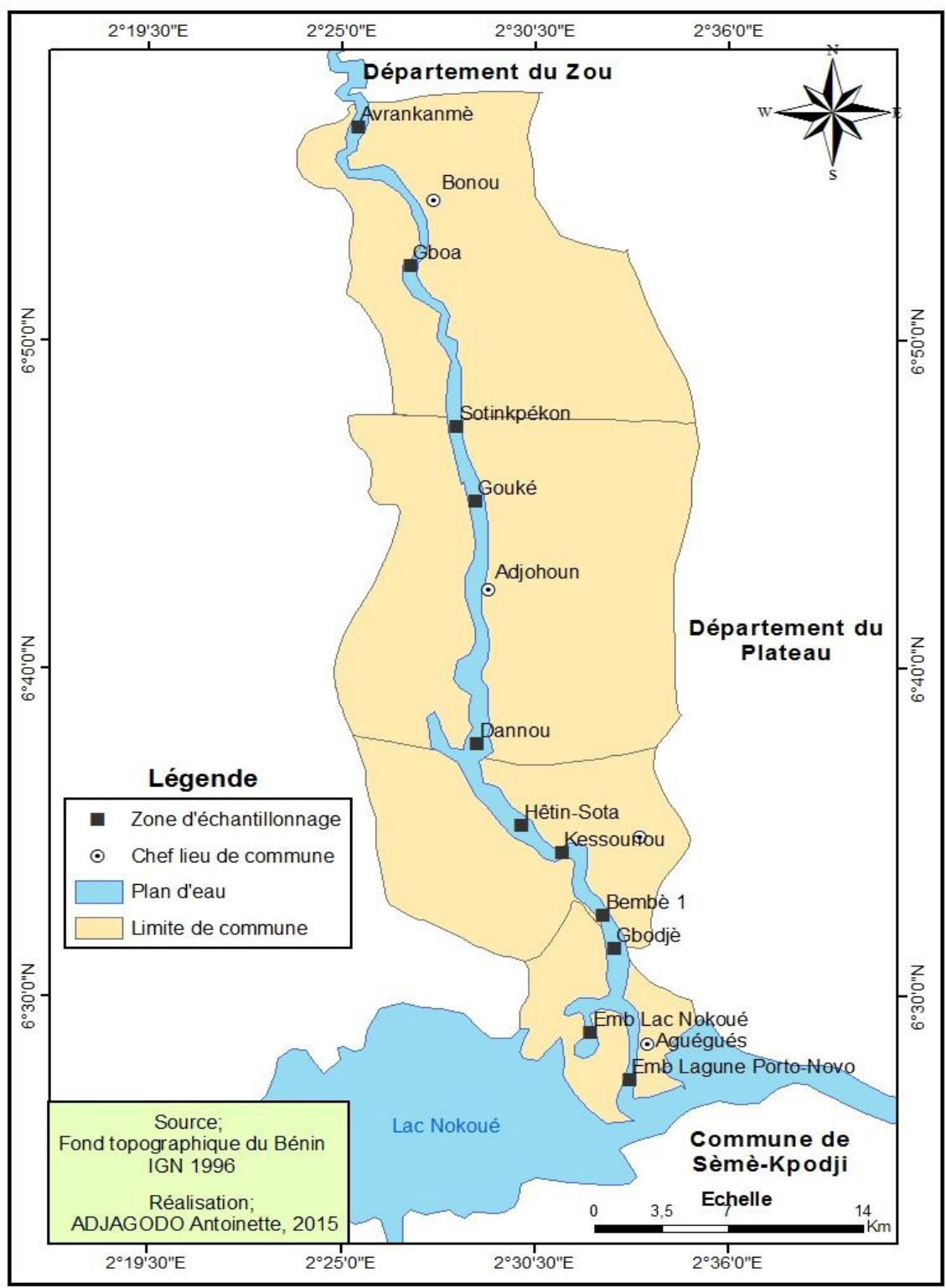

Figure 2 : Carte de localisation des points de prélèvement

\section{Détermination des paramètres physiques}

Les paramètres physiques tels que le potentiel hydrogène, l'oxygène dissous, la turbidité et les matières en suspension ont été mesurés in situ sur 88 échantillons d'eau prélevés (pour les deux campagnes d'échantillonnage) au niveau du fleuve de la Basse Vallée de l'Ouémé. Le $\mathrm{pH}$ et l'oxygène dissous ont été mesurés à l'aide du pH /Oxi mètre WTW 340i. Les matières 
en suspension et la turbidité ont été mesurées grâce au colorimètre DR890/HACH.

\section{Détermination des germes de contamination fécale}

Les germes de contamination fécale tels que les Coliformes totaux (CT), les Coliformes thermotolérants (Cth), Escherichia coli, les Entérocoques fécaux (EF) ont été recherchés dans les échantillons d'eau prélevés au niveau du fleuve de la Basse Vallée de l'Ouémé. La méthode utilisée pour la détermination de ces germes est la technique de filtration sur membrane (tableau 1).

Tableau 1 : Méthodes de recherche et d'identification des germes de contamination fécale

\begin{tabular}{|c|c|c|c|c|}
\hline $\begin{array}{l}\text { Germes et } \\
\text { références de la } \\
\text { méthode }\end{array}$ & $\begin{array}{c}\text { Types } \\
\text { d'ensemencement }\end{array}$ & $\begin{array}{l}\text { Milieux de } \\
\text { culture }\end{array}$ & $\begin{array}{l}\text { Conditions } \\
\text { de culture }\end{array}$ & $\begin{array}{c}\text { Colonies } \\
\text { caractéristiques }\end{array}$ \\
\hline $\begin{array}{l}\text { Coliformes totaux } \\
\text { (NFV-066/1996) }\end{array}$ & \multirow{4}{*}{$\begin{array}{l}\text { Filtration de } 100 \\
\text { mL d'eau sur } \\
\text { membrane } \\
(0,45 \mu \mathrm{m})\end{array}$} & \multirow{3}{*}{$\begin{array}{c}\text { Gélose } \\
\text { Violet Red } \\
\text { Bile } \\
\text { Lactose } \\
\text { Agar } \\
\text { (VRBL) }\end{array}$} & $\begin{array}{l}24-48 \\
\text { heures } \\
\text { à } 37^{\circ} \mathrm{C}\end{array}$ & $\begin{array}{l}\text { Colonies } \\
\text { violacées }\end{array}$ \\
\hline $\begin{array}{c}\text { Coliformes } \\
\text { thermotolérants } \\
(\mathrm{NFV}-066 / 1996)\end{array}$ & & & \multirow[t]{2}{*}{$\begin{array}{c}24-48 \\
\text { heures à } \\
44^{\circ} \mathrm{C}\end{array}$} & $\begin{array}{l}\text { Colonies } \\
\text { violacées }\end{array}$ \\
\hline $\begin{array}{c}\text { E. coli }(\mathrm{NFV}- \\
066 / 1996)\end{array}$ & & & & $\begin{array}{l}\text { Colonies rouge } \\
\text { violacées }\end{array}$ \\
\hline $\begin{array}{c}\text { Entérocoques } \\
\text { fécaux AFNOR } \\
\text { NF ISO 7899-2 } \\
(2000)\end{array}$ & & $\begin{array}{l}\text { Gélose } \\
\text { Slanetz } \\
\text { Bartley }\end{array}$ & $\begin{array}{l}24-48 \\
\text { heures } \\
\text { à } 37^{\circ} \mathrm{C}\end{array}$ & $\begin{array}{c}\text { colonies } \\
\text { rouges/violettes/ } \\
\text { roses }\end{array}$ \\
\hline
\end{tabular}

\section{Analyse statistique}

Les valeurs des paramètres physiques et bactériologiques déterminées ont fait l'objet d'une analyse statistique descriptive (moyenne, minimum et maximum). L'Analyse en Composante Principale (ACP) a été également réalisée afin de mettre en évidence les relations entre les paramètres physiques et bactériologiques des eaux du fleuve Ouémé et leur distribution dans les différents sites échantillonnés. Le logiciel STATISTICA version 9 a été utilisé pour la réalisation de l'ACP.

\section{Résultats}

Les résultats des paramètres physiques varient d'une saison à une autre et selon les échantillons. 


\section{Qualité physique des eaux échantillonnées du fleuve Ouémé} Potentiel hydrogène

Les valeurs de $\mathrm{pH}$ mesurées (figure 3) pendant la période de basses et hautes eaux varient respectivement de 7,1 $\pm 0,01$ à $7,27 \pm 0,01$; $6,67 \pm 0,01$ à $6,97 \pm 0,01$. Les écarts types oscillent entre 0,01 et 0,05 . La plus grande valeur est obtenue à Gbodjè pendant la période de basses eaux et la plus petite valeur à Dannou pendant la période de hautes eaux. Les eaux analysées pendant la période de basses eaux sont faiblement basiques par contre celles de la période de hautes eaux sont presque neutre ( $\mathrm{pH}$ proche de 7).

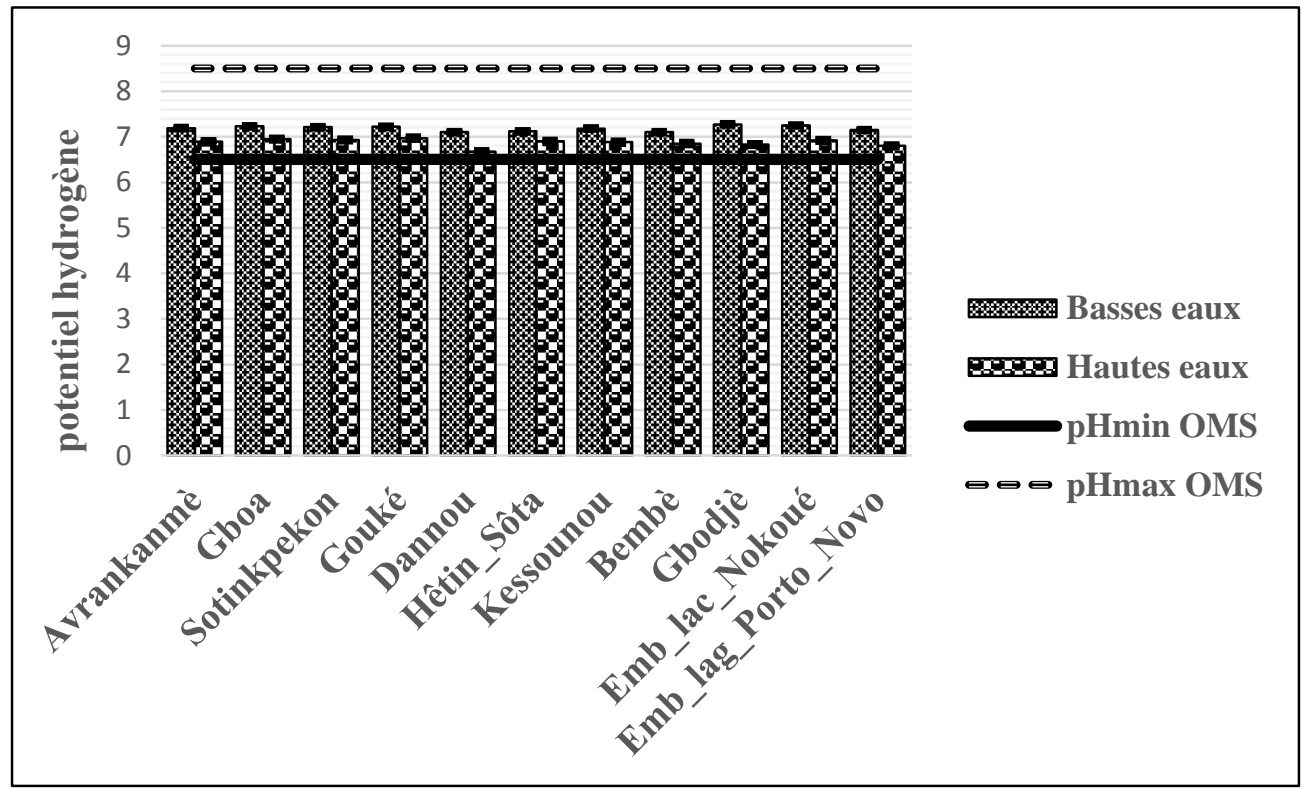

Figure 3 : Variation saisonnière du pH mesuré dans les eaux du fleuve Ouémé

\section{Oxygène dissous}

Les valeurs de l'oxygène dissous (figure 4) obtenues dans les eaux prélevées pendant les deux saisons varient de 2,49 $\pm 0,01 \mathrm{mg} / \mathrm{L}$ à 12,46 \pm $0,01 \mathrm{mg} / \mathrm{L}$. Les écarts types oscillent entre 0,01 et 0,09 . La plus forte valeur est enregistrée pendant la période de hautes eaux au niveau de l'embranchement de la lagune de Porto- Novo et la plus petite valeur est obtenue pendant la période de basses eaux au niveau de l'embranchement du lac Nokoué. 


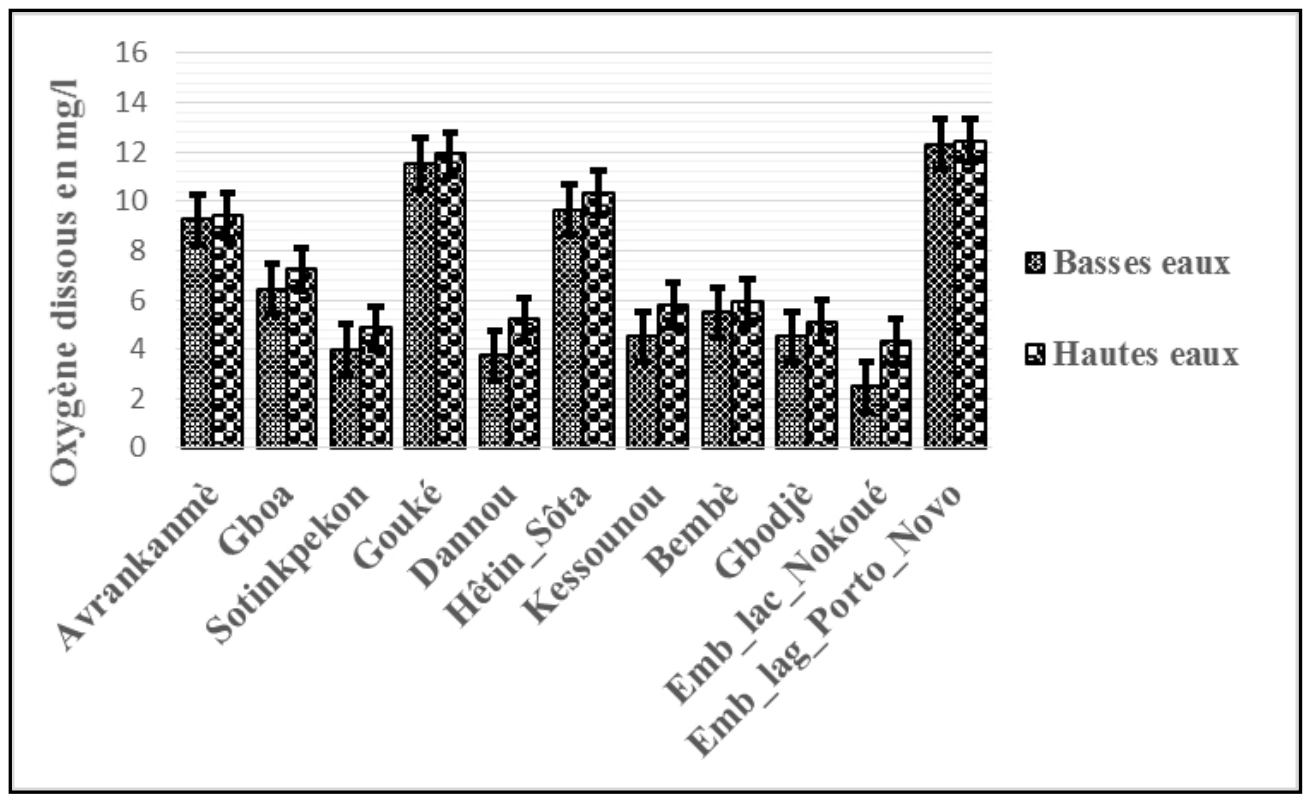

Figure 4 : Variation saisonnière de l'oxygène dissous mesuré dans les eaux du fleuve Ouémé

\section{Turbidité}

Les valeurs de la turbidité (figure 5) obtenues dans les échantillons d'eau prélevés pendant les deux saisons varient de 13,91 $\pm 0,01$ $\mathrm{mg} / \mathrm{L}$ à $158 \pm 0,5 \mathrm{mg} / \mathrm{L}$. Les écarts types varient de 0,01 à 0,08 . L'eau la plus trouble est obtenue à Gboa en période de basses eaux et la moins trouble à Hêtin-Sota en période de hautes eaux.

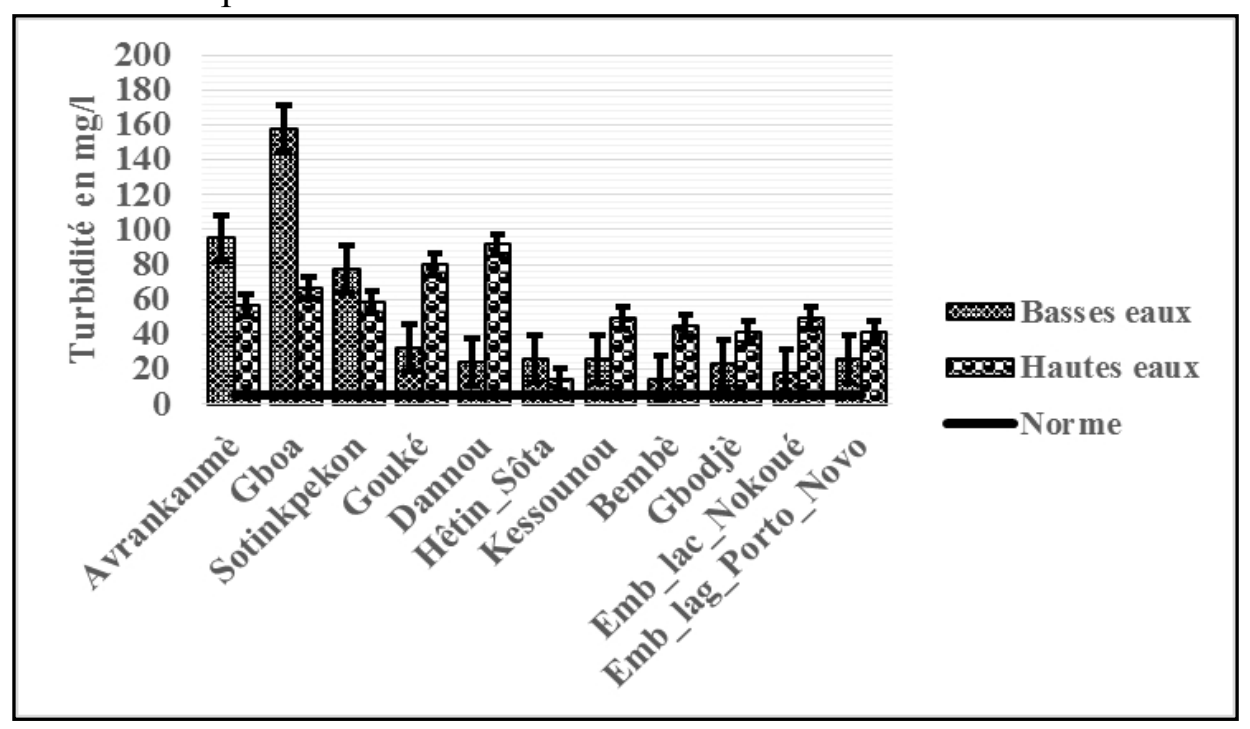

Figure 5: Variation saisonnière de la turbidité mesurée dans les eaux du fleuve Ouémé 


\section{Matière en suspension}

Les concentrations moyennes des matières en suspension (figure 6) mesurées pendant la période de basses et hautes eaux varient respectivement de $8 \pm 0,5 \mathrm{mg} / \mathrm{L}$ à $118 \pm 0,5 \mathrm{mg} / \mathrm{L}$ et de $11,32 \pm 0,01 \mathrm{mg} / \mathrm{L}$ à $39,32 \pm 0,01 \mathrm{mg} / \mathrm{L}$. Les écarts types oscillent entre 0,01 et 0,5 . La plus grande valeur est obtenue à Gboa pendant la période de basses eaux et la plus petite valeur à Bembè pendant cette même période.

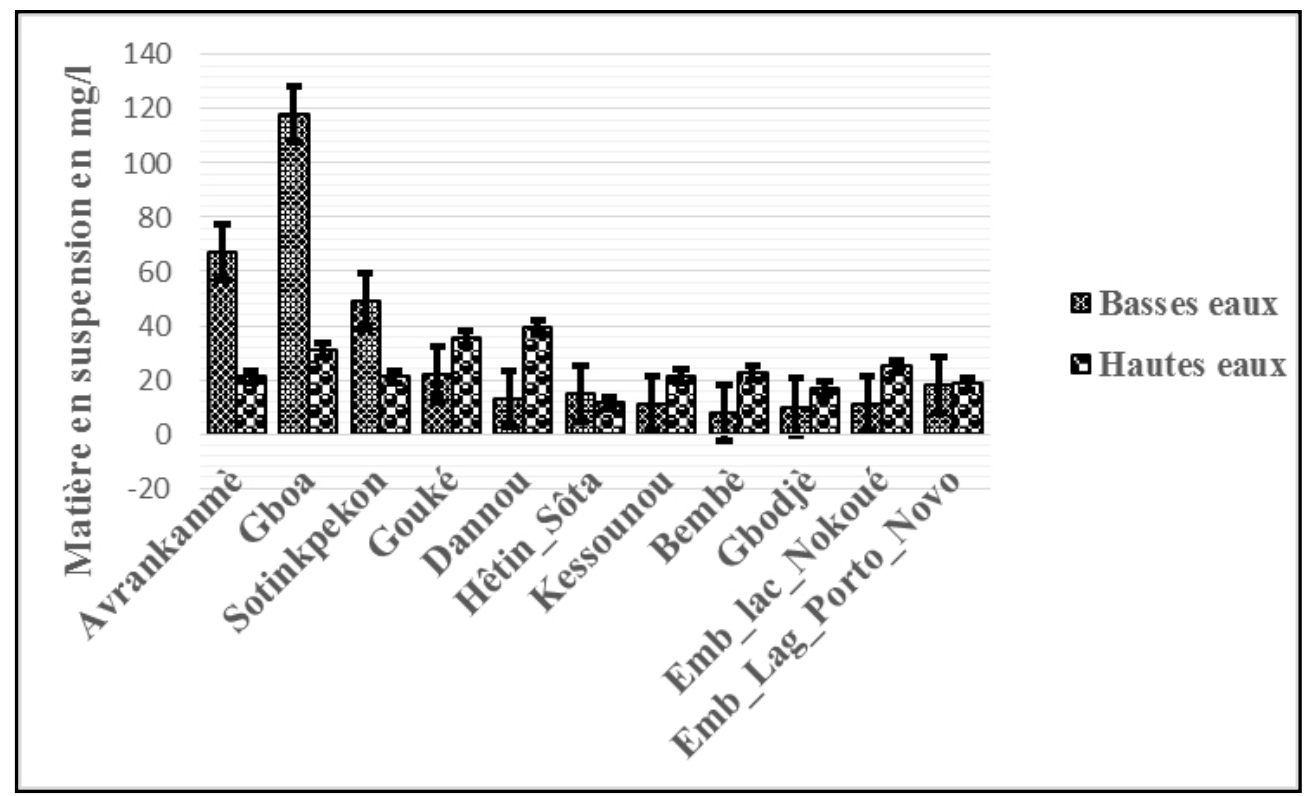

Figure 6: Variation saisonnière de la matière en suspension mesurée dans les eaux du fleuve Ouémé

\section{Qualité bactériologique des eaux échantillonnées du fleuve Ouémé}

Les résultats de la recherche des germes indicateurs de pollution fécale montrent que les eaux analysées pendant les périodes de basses et de hautes eaux contiennent des taux divers de Coliformes totaux, de Coliformes thermotolérants et $E$. coli. Les Entérocoques fécaux sont absents dans les eaux analysées pendant les deux saisons d'échantillonnage.

\section{Coliformes totaux}

Les résultats des analyses bactériologiques des échantillons d'eau du fleuve de la Basse Vallée de l'Ouémé révèlent des charges bactériennes en Coliformes totaux (figure 7) qui varient de $0,6.10^{2} \pm 1 \mathrm{UFC} / 100 \mathrm{~mL}$ à $16,1.10^{2} \pm 2 \mathrm{UFC} / 100 \mathrm{~mL}$ avec une moyenne de $2,57.10^{2} \pm 451 \mathrm{UFC} / 100$ $\mathrm{mL}$ pendant la période de basses eaux et de $1,20.10^{2} \pm 2 \mathrm{UFC} / 100 \mathrm{~mL}$ à $17,1.10^{2} \pm 2$ UFC/100 mL avec une moyenne de $3,35.10^{2} \pm 459 \mathrm{UFC} / 100$ $\mathrm{mL}$ pendant la période de hautes eaux. Les écarts types oscillent entre 1 et 3 . 
Les fortes charges en Coliformes totaux pendant les deux saisons sont obtenues à Gouké alors que les plus faibles charges sont obtenues à Avrankanmè pendant la période de basses eaux et à Bembè pendant la période de hautes eaux.

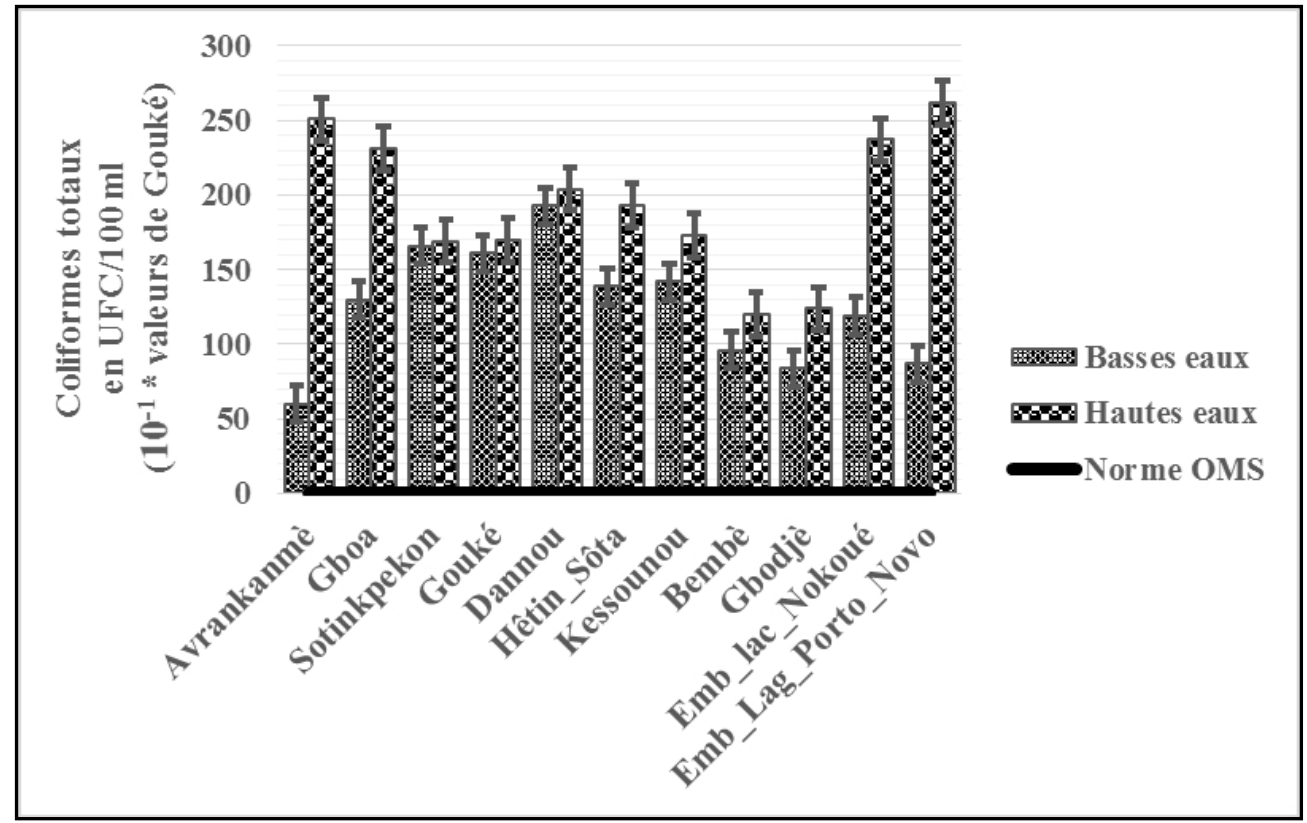

Figure 7 : Variation saisonnière des charges en Coliformes totaux dans les échantillons d'eau du fleuve Ouémé

\section{Coliformes thermotolérants}

Les charges en Coliformes thermotolérants (figure 8) dénombrées dans l'eau du fleuve pendant la période de basses eaux varient de $0,18 \cdot 10^{2} \pm$ $1 \mathrm{UFC} / 100 \mathrm{~mL}$ à $10,9.10^{2} \pm 2 \mathrm{UFC} / 100 \mathrm{~mL}$ avec une moyenne de $1,42.10^{2} \pm$ $315 \mathrm{UFC} / 100 \mathrm{~mL}$. Celles dénombrées dans l'eau du fleuve pendant la période de hautes eaux varient de $7 \cdot 10^{1} \pm 1 \mathrm{UFC} / 100 \mathrm{~mL}$ à $13 \cdot 10^{2} \pm 3$ UFC/100 mL avec une moyenne de $2,24.10^{2} \pm 359 \mathrm{UFC} / 100 \mathrm{~mL}$. Les écarts types oscillent entre 1 et 3 . Les fortes charges en Coliformes thermotolérants dénombrées dans l'eau du fleuve pendant les deux saisons sont obtenues à Gouké. Les plus faibles charges sont enregistrées au niveau de l'embranchement de la lagune de Porto-Novo pendant la période de basses eaux et à Sotinkpékon pendant la période de hautes eaux. 


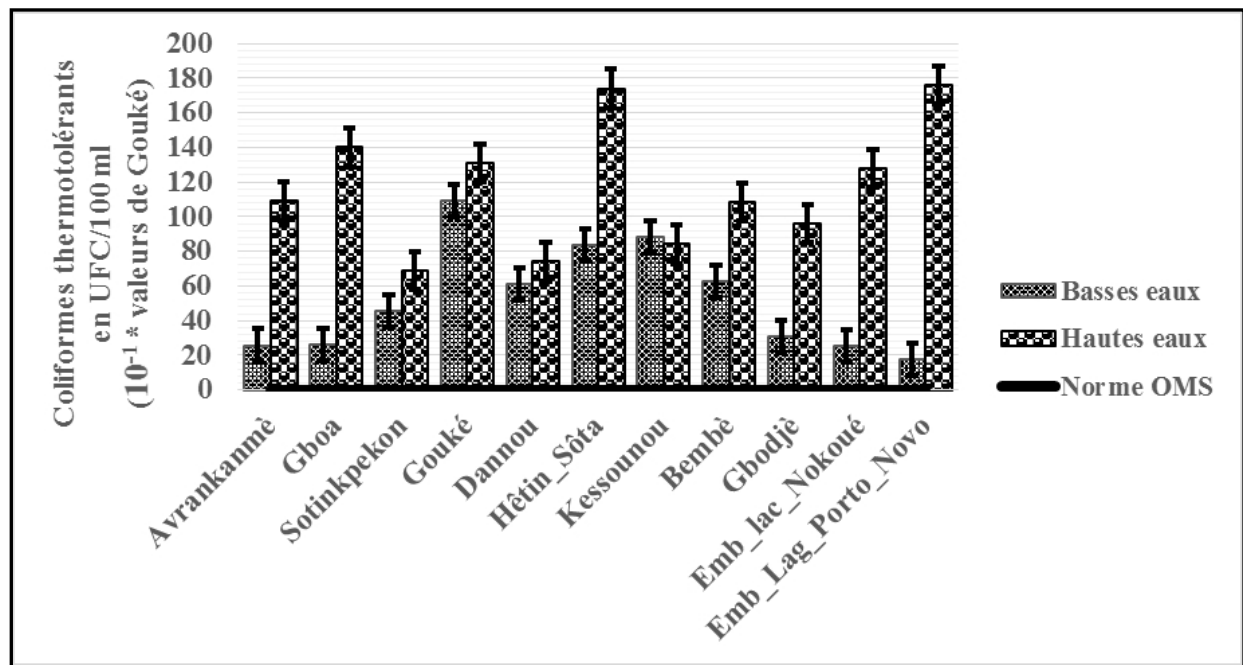

Figure 8 : Variation saisonnière des charges en Coliformes thermotolérants dans les échantillons d'eau du fleuve Ouémé

\section{Escherichia coli}

Les charges bactériennes moyennes en $E$. coli pendant la période de basses et hautes eaux varient respectivement de $10^{1} \pm 1 \mathrm{UFC} / 100 \mathrm{~mL}$ et $7,3.10^{2} \pm 2 \mathrm{UFC} / 100 \mathrm{~mL} ;$ de $1,8.10^{1} \pm 2 \mathrm{UFC} / 100 \mathrm{~mL}$ et $9.10^{2} \pm 2 \mathrm{UFC} / 100$ $\mathrm{mL}$. Les écarts types varient de 1 à 2 . La plus grande charge est obtenue à Gouké pendant la période de hautes eaux et la plus petite charge au niveau de l'embranchement du lac Nokoué pendant la période de basses eaux.

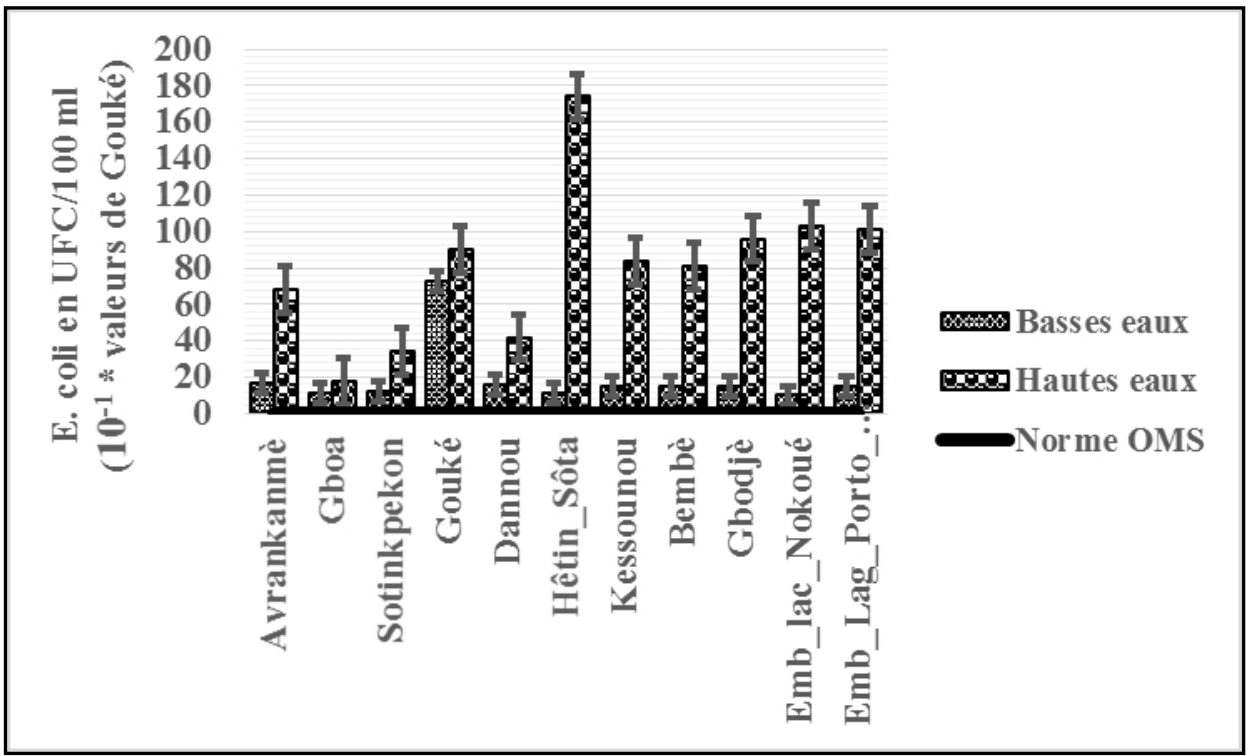

Figure 9 : Variation saisonnière des charges en E. coli dans les échantillons d'eau du fleuve Ouémé 


\section{Analyse en composante principale}

Les résultats de l'analyse en composantes principales des paramètres physiques et bactériologiques obtenus révèlent que les deux premiers axes expliquent à $70,36 \%$ toute la variabilité de l'influence des paramètres physiques et bactériologiques sur l'eau du fleuve. La projection des variables sur le plan factoriel F1-F2 (figure 10) montre que les Coliformes totaux, les Coliformes thermotolérants, E. coli et le l'oxygène dissous sont corrélés négativement avec l'axe F1; la turbidité corrélée positivement avec l'axe F2 et les matières en suspension corrélées négativement avec l'axe F2. L'analyse de la projection des individus sur le plan factoriel F1-F2 (figure 11) nous permet de mettre en évidence trois regroupements. Le premier regroupement prend en compte la localité de Gouké qui a une eau oxygénée très chargée en Coliformes totaux, en Coliformes thermotolérants et en $E$. coli. Le deuxième regroupement prend en compte les localités Gboa, Sotinkpekon et Avrankanmè qui ont des eaux troubles moins chargées en Coliformes totaux, en Coliformes thermotolérants et en $E$. coli. Le troisième regroupement prend en compte les localités de Dannou, de Gbodjè, de Bembè, de Kessounou, de Hêtin-Sota, des embranchements du lac Nokoué et de la lagune de Porto Novo qui ont des eaux très riches en matières en suspension.

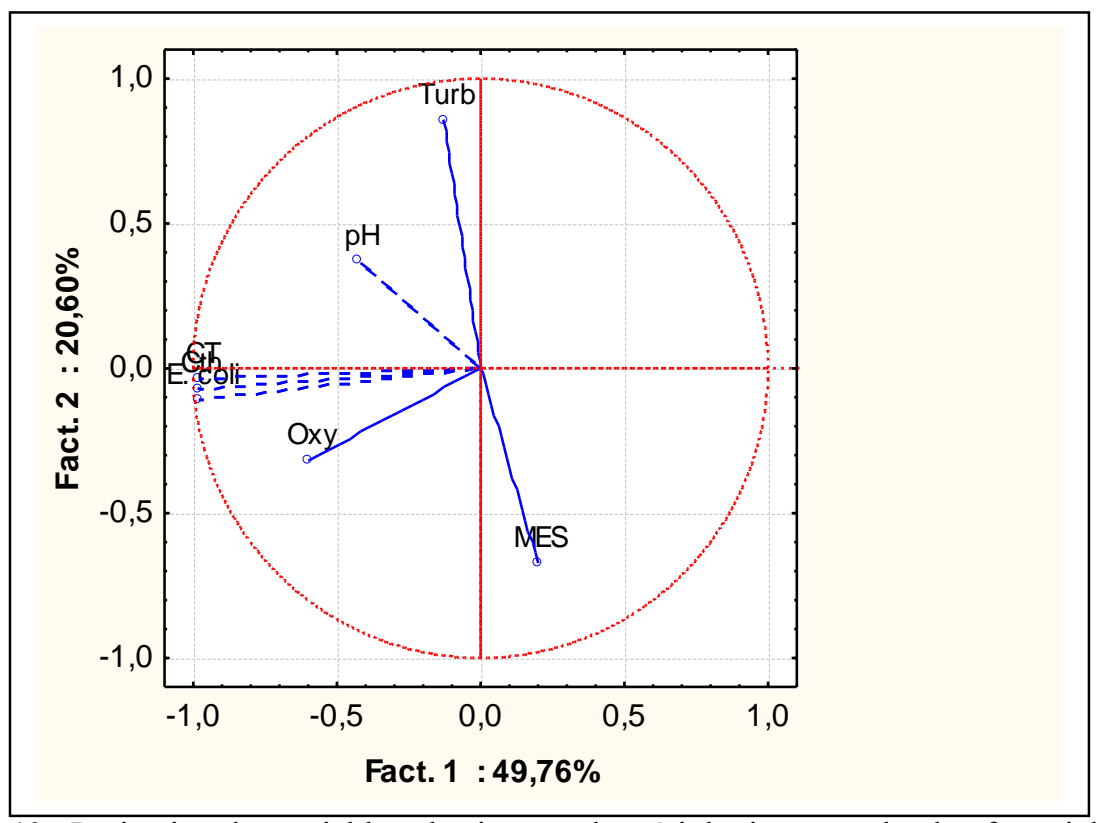

Figure 10 : Projection des variables physiques et bactériologiques sur le plan factoriel F1-F2 


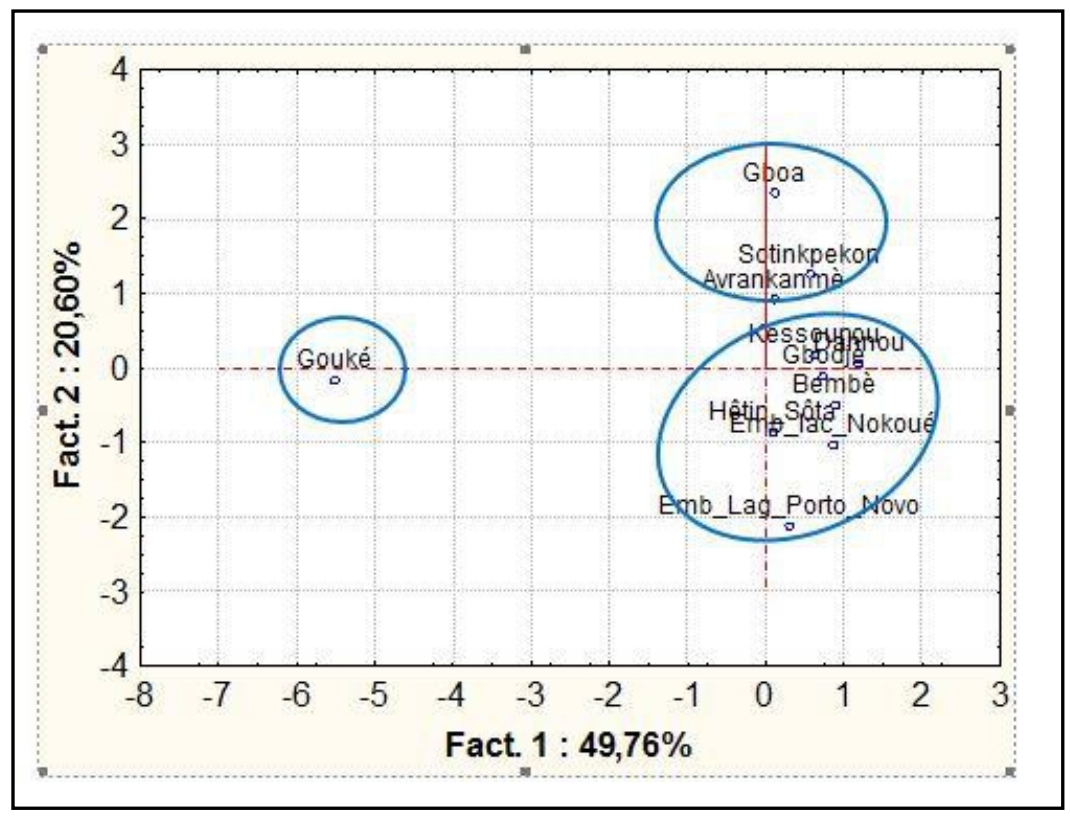

Figure 11 : Projection des individus (localités de prélèvement) sur le plan factoriel

\section{Discussion}

Les résultats des analyses physiques des échantillons d'eau du fleuve prélevés dans la Basse Vallée de l'Ouémé révèlent que le $\mathrm{pH}$ quel que soit la période de l'échantillonnage est conforme aux critères normatifs de l'OMS $(6,5 \leq \mathrm{pH} \leq 8,5)$. Les teneurs en oxygène dissous mesurées pendant les deux périodes d'échantillonnage sont supérieures à $3 \mathrm{mg} / \mathrm{L}$ d' $\mathrm{O}_{2}$ sauf pour la période de basses eaux au niveau de l'embranchement du lac Nokoué. Selon Beaux (1998), cette teneur en oxygène dissous observée dans l'eau du fleuve pendant cette période qualifie cette zone de polluée car la concentration en oxygène dissous dans cette zone est inférieure à $3 \mathrm{mg} / \mathrm{L} \mathrm{d}^{\prime} \mathrm{O}_{2}$. Les valeurs de $\mathrm{pH}$ qui oscillent de 6,37 à 7,27 sont bonnes en aquaculture selon Abou (2001), si les valeurs des pH sont comprises entre 6,5 à 9. Les valeurs maximales des MES et de la turbidité respectivement $118 \mathrm{mg} / \mathrm{L}$ et $158 \mathrm{NTU}$ observées à Gboa traduisent l'influence de l'exploitation de sable pendant la période de basses eaux sur l'eau du fleuve Ouémé.

Les résultats des analyses bactériologiques effectuées présentent une grande diversité bactérienne du point de vue quantitative et qualitative durant les deux périodes (basses et hautes eaux). Cette variation s'observe également au niveau d'un même point de prélèvement. La charge bactérienne en Coliformes totaux, en Coliformes thermotolérants et en $E$. coli présente des fluctuations irrégulières de l'amont vers l'aval, avec des charges maximales respectives de $1,71.10^{3} \pm 2 \mathrm{UFC} / 100 \mathrm{~mL} ; 1,3.10^{3} \pm 3$ 
UFC/100 mL et $9.10^{2} \pm 2$ UFC/100 mL obtenues au niveau de Gouké. Les charges bactériennes dénombrées dans les échantillons d'eau du fleuve prélevés pendant les deux périodes sont hors normes selon les critères normatifs de l'Organisation Mondiale de la Santé (OMS, 2011). La présence des Coliformes totaux et thermotolérants dans ces eaux peut être due aux rejets des eaux usées domestiques et à ses usages pour la baignade. Selon Manizan et al. (2010), le déversement des eaux usées domestiques sans aucun traitement préalable peut participer à la contamination des eaux de surface. D'après Jacinta et al. (2007), la qualité des eaux de surface de nombreux lacs et rivières est sujet de la présence de niveaux élevés en Coliformes thermotolérants. La contamination de l'eau du fleuve Ouémé par ce germe peut être expliquée par la présence d'une contamination provenant principalement des déchets fécaux d'origine humaine (déchets domestique) et animal (effluents d'élevage) ainsi que de l'installation des latrines publiques sur le fleuve. L'utilisation des matières fécales des animaux (bouses de bœufs et de lapins, fientes de volailles) comme engrais dans l'agriculture dans la Basse Vallée de l'Ouémé est aussi source de contamination fécale. La présence des Coliformes thermotolérants dans l'eau constitue un bon indice de pollution surtout attribuée à une contamination fécale (Ladjel, 2009). Selon Patoine (2011) et Leclerc (1981), les Coliformes thermotolérants sont abondants dans les fèces et représentent les indicateurs prédominants de l'environnement. E. coli est aussi détecté dans $100 \%$ des échantillons d'eau du fleuve prélevés pendant les deux périodes. En ce qui concerne E. coli leur présence en nombre élevé quelle que soit la période au niveau de Gouké, Hêtin-sota, Gbodjè, de Kessounou, des embranchements du lac Nokoué et de la lagune de Porto-Novo indique que l'eau du fleuve est vulnérable. Cette déclaration est liée au fait que ce germe est le chef de fil des pathogènes (Agassounon et al., 2014). Par ailleurs, la détection d'E. coli dans les échantillons d'eau prélevés est considérée comme suffisante pour affirmer la nature fécale de la pollution (Aboulkacem, 2007). En effet, E. coli constitue le seul membre du groupe des Coliformes totaux que l'on trouve exclusivement dans les matières fécales des humains et des animaux. Leur présence dans l'eau indique non seulement une contamination récente par des matières fécales, mais aussi la présence possible de bactéries, virus et protozoaires pathogènes (INSPQ, 2003 ; John et Donald, 2010). L'absence des Entérocoques fécaux dans les eaux analysées est observée pendant les deux périodes. Cette absence confirme que la contamination de l'eau du fleuve par les matières fécales est récente car la présence des Entérocoques fécaux dans l'eau indique une contamination ancienne.

La différence des charges bactériennes enregistrées pendant les deux périodes où les fortes charges sont observées en saison pluvieuse peuvent être dues au $\mathrm{pH}$ enregistré au niveau de ces eaux en ces périodes. Les eaux 
échantillonnées en période de basses eaux ont un $\mathrm{pH}$ faiblement basique et en période de hautes eaux un $\mathrm{pH}$ presque neutre (proche de 7). D'après Mayo (1995) et N'Diaye et al. (2011), les pH basiques peuvent entrainer une nette diminution de la survie des germes fécaux. Par contre, le caractère presque neutre du $\mathrm{pH}$ peut favoriser le développement de bons nombres de bactéries, notamment les Coliformes totaux, les Coliformes thermotolérants et les Streptocoques fécaux dans les eaux fluviales (Bury-Moné, 2007 ; Manizan et al., 2010). De même, cette forte charge en ces germes dénombrés au cours de la période de hautes eaux peut être aussi due au phénomène de ruissellement qui entraîne tous les déchets solides (déjections humaines et animales) et liquides (eaux usées) vers l'eau du fleuve. Des observations similaires ont été faites par Bahhou (1991) au niveau de certaines zones du littoral méditerranéen Nord-Ouest de Maroc, par Zegmout et al. (2011) au niveau de la zone côtière Saidia/Moulouya de la région orientale du Maroc et par Dovonou et al. (2011) au niveau du lac Nokoué au Bénin. L'abondance des germes fécaux pendant la saison des pluies peut essentiellement être due à une majoration des apports anthropiques par le lessivage des sols souillés et par la vidange des égouts et aux eaux de ruissellement (Hunter, 2003 ; Fouad et al., 2013 ; Ouhmidou et al., 2015). Les travaux réalisés par Kambire et al. (2012) ont montré que la saison des pluies et celle des crues favorisent l'enrichissement des eaux lagunaires en éléments nutritifs utiles à la prolifération des bactéries. Une forte turbidité peut aussi permettre à des micro-organismes de se fixer sur les particules en suspension, donc la qualité bactériologique d'une eau turbide est donc suspecte (Ghazali et Zaid, 2013).

Les résultats de l'analyse en composante principale montrent que la localité de Gouké a enregistré des valeurs élevées en oxygène dissous, en Coliformes totaux, en Coliformes thermotolérants et en E. coli dans l'eau du fleuve. Cette analyse indique que dans cette localité l'eau est fortement polluée par les germes de contamination fécale et que la pollution est liée à l'activité domestique qui se fait au bord du fleuve et au lessivage des champs agricoles. Par contre, dans les localités de Avrankanmè, de Sotinkpekon et de Gboa les eaux prélevées sont très troubles. Cette pollution est liée à l'exploitation de sable, au transport des hydrocarbures (projection des tonneaux d'essence dans l'eau du fleuve pour son transport d'une rive à l'autre) et à la traversée des motos par voie d'eau en période de basses eaux. Il ressort également de l'ACP que dans les localités de Dannou, de HêtinSota, de Bembè, de Gbodjè, des embranchements du lac Nokoué et de la lagune de Porto-Novo l'eau est très riche en matières en suspension et que la pollution de l'eau du fleuve est liée à l'activité domestique (lessive, baignade, vaisselle, etc.) et aussi à l'exploitation de sable. En définitif, la pollution anthropique du fleuve est liée aux rejets des ordures ménagères et 
des eaux usées, de la baignade, de la lessive, du transport des produits pétroliers sur le fleuve, de l'exploitation du sable, du lessivage des champs agricoles et des apports des polluants venus de l'amont de notre zone d'étude.

\section{Conclusion}

Cette étude a permis de mettre en évidence l'état de pollution physique et bactériologique de l'eau du fleuve de la Basse Vallée de l'Ouémé. Les résultats issus de cette étude révèlent que les eaux de ce fleuve sont troubles et polluées par les germes de contamination fécale (Coliformes totaux, Coliformes thermotolérants, $E$. coli). Une absence totale de Streptocoques fécaux est notée dans $100 \%$ des échantillons d'eau analysés pendant les deux campagnes d'échantillonnage. Les germes de contamination fécale dénombrés dans l'eau du fleuve sont dus à des rejets des eaux usées domestiques, à la défécation dans l'eau du fleuve, à la lessive et à la vaisselle au bord du fleuve. Il est nécessaire de poursuivre des études sur le site afin de bien repérer les causes réelles d'une telle pollution très accentuée.

\section{References:}

1. Abou Y., 2001. Effet d'une couverture d'Azolla LAM sur la production piscicole du tilapia Oreochromis niloticus (L.) en étangs dans la banlieue de Cotonou (Bénin). Mémoire présenté pour l'obtention du diplôme d'études complémentaire en éco-technologie des eaux continentales. Facultés Universitaires Notre Dame de la Paix. Faculté des Sciences. Namur-Belgique.

2. Aboulkacem A., Chahlaoui A., Soulaymani A., Rhazi-Filali F., Benali D., 2007. Etude comparative de la qualité bactériologique des eaux des Oueds Boufekrane et Ouislane à la traversée de la ville de Meknès (Maroc). Rev. Microbiol. Ind. San et Environn. 1 : 10 - 22.

3. Adjagodo A., Agassounon Djikpo Tchibozo M., Kelomè N.C., Lawani R., 2016. Flux des polluants liés aux activités anthropiques, risques sur les ressources en eau de surface et la chaine trophique à travers le monde : synthèse bibliographique. Int. J. Biol. Chem. Sci. 10 (3) : 1459-1472.

4. Afri-Mehennaoui F.Z., Sahli L., Mehennaoui S., 2009. Evaluation de la contamination par le cadmium, le plomb et le zinc de l'eau, des sédiments de l'Oued Rhumel et son affluent le Boumerzoug, et leur transfert vers une plante semi-aquatique : Rorippa nasturtiumaquaticum (L.). Sciences \& Technologie. 29: 45-55.

5. Agassounon Djikpo Tchibozo M., Tadjou A., Anago D.G., Dovonou E.F., Ayi-Fanou L., 2014. Qualité physico-chimique et 
bactériologique des eaux de boisson dans les arrondissements de la commune de Kétou au Bénin. Microbiol. Ind. San. Environ., 8: 187207.

6. Aguiza A.E., Ombolo A., Ngassoum M.B., Mbawala A., 2014. Suivi de la qualité physico-chimique et bactériologique des eaux des cours d'eau de N'Gaoundéré, au Cameroun. Afrique science. 10(4): 135 145

7. Bagalwa M., 2013. Estimation of pollution loading into Lake Kivu. Case of Kahuwa micro-catchment in DRCongo. Thesis of Master, UEA, Bukavu, 71p.

8. Bahhou J., 1991. Contribution à l'étude bactériologique des eaux marines littorales du Nord du Maroc Thèse de doctorat de $3^{\text {ème }}$ cycle, Université Mohammed V Rabat, 127 p.

9. Beaux J.-F., 1998. L'environnement, Repères pratiques, Nathan, 160 $P$.

10. Dovonou F., Aïna M., Boukari M., Alassane A., 2011. Pollution physico-chimique et bactériologique d'un écosystème aquatique et ses risques écotoxicologiques : cas du lac Nokoué au Sud Benin. Int. J. Biol. Chem. Sci. 5(4): 1590-1602.

11. Errochdi S., El Alami M., Bennas N., Belqat B., Ater M., Fdil F., 2012. Etude de la qualité physicochimique et microbiologique de deux réseaux hydrographiques nord marocains: Laou et Tahaddart. Méditerranée. 118: 41-51. URL : http://mediterranee.revues.org/6221

12. Fouad S., Cohen N., Hajjami K., Chlaida M., 2013. Qualité physicochimique et contamination métallique des eaux de l'Oued Hassar: impacts des eaux usées de la localité de Mediouna (Periurbain de Casablanca, Maroc). ScienceLib Editions Mersenne : Volume 5, $\mathrm{N}^{\circ}$ 130113, ISSN 2111-4706.

13. Ghazali D., Zaid A., 2013. Etude de la qualité physico-chimique et bactériologique des eaux de la source Ain Salama-Jerri (Région de Meknès - Maroc). Larhyss journal, ISSN 1112-3680, N 12, pp25-36

14. Hebert S., Legare S., 2000. Suivi de la qualité des rivières et petits cours d'eau, Direction du suivi de l'état de l'environnement, ministère de 1'Environnement, Québec, envirodoq No ENV-20010141, 3 annexes rapport $\mathrm{N}^{\circ} \mathrm{qE}-123,24 \mathrm{p}$.

15. Hunter P.R., 2003. Principles and components of surveillance systems. In P. R. Hunter, M. Waite, \& E. Ronchi (Eds.), Drinking water and infectious disease: Establishing the links. Boca Raton, FL: CRC Press.

16. INSPQ (Institut National de la Santé Publique du Québec)., 2003. Fiche Coliformes totaux Fiches synthèses sur l'eau potable et la santé humaine. Groupe scientifique, Décembre 2004, 4p. 
17. Jacinta C., Uzoigwe E., O’Brien H., Brown E.J., 2007. Using nutrient utilization patterns to determine the source of Escherichia coli found in surface water. African Journal of Environmental Science and Technology. 1(1): 007-013.

18. John P., Donald A., 2010. Microbiologie, 3ème Édition, 1216 p.

19. Kambire O., Adingra A.A., Kakou C.A., Koffi-Nevry R., 2012. Indicateurs de pollution fécale dans une lagune tropicale à forte influence continentale (lagune Aby, Côte d'ivoire). Agronomie Africaine. 24 (2) : 89 - 100.

20. Kazi T.G., Arain M.B., Jamali M.K., Jalbani N., Afridi H.I., Sarfraz R.A., Baig J.A., Shah A.Q., 2009. Assessment of water quality of polluted lake using multivariate statistical techniques: A case study. Ecotoxicology and Environmental Safety. 72 : 301-309.

21. Leclerc H.F., 1981. Les indicateurs bactériens dans le contrôle bactériologique de l'eau : exigences et limites. J. Fr. hydrol. $35: 213$ $-228$.

22. Manizan N.P., Ouattara A., Gourene G., Dosso M., 2010. Influence des caractéristiques physico-chimiques sur la distribution spatiotemporelle des densités bactériennes dans le système fluvio-lacustre de la Bia, Sud-Est de la Côte d'ivoire. Rev. Ivoir. Sci. Technol., 15: $201-210$.

23. Mayo A.W., 1995. Modelling coliform mortality in waste stabilization ponds. J. Environ. Engineer, 121 (2): 140-152.

24. MONIOD F., 1973. Régime hydrologique de I'Ouémé (Dahomey). Cah. O.R.S.T.O.M., sbr. Hydrol.

25. N'Diaye A.D., Kankou M.O.S.A.O., Lo B., 2011. Contribution de l'ACP et les paramètres physiques dans l'évaluation des Coliformes Fécaux contenus dans les effluents de la STEP du périmètre maraicher de Sebkha, Nouakchott. ScienceLib Editions Mersenne : 3 (111113) ISSN 2111-4706

26. Ndahama N., Bagalwa M., Bayongwa C., 2014. Etude de la pollution organique totale et fécale dans les systèmes aquatiques de l'Est de la République Démocratique du Congo. Afrique Science 10(2) : 328 337.

27. Ouhmidou M., Chahlaoui A., Kharroubi A., Chahboune M., 2015. Etude de la qualité physico-chimique et bactériologique des eaux du barrage Hassan Addakhil d'Errachidia (Maroc). J. Mater. Environ. Sci. 6 (6) : 1663-1671.

28. OMS (Organisation Mondiale de la Santé)., 2011. Guidelines for drinking-water quality. Fourth edition. 
29. Patoine M., 2011. Influence de la densité animale sur la concentration des coliformes fécaux dans les cours d'eau du Québec méridional, Canada, Revue des sciences de l'eau, 24 (4) : 421-435.

30. Saab H.B., Nassif N., El Samrani A.G., Rosette D., Medawar S., Ouaïni N., 2007. Suivi de la qualité bactériologique des eaux de surface (rivière Nahr Ibrahim, LIBAN). Revue des Sciences de l'Eau. $20(4): 341-352$.

31. WHO (world health organization)., 2017. Don't pollute my future! The impact of the environment on children's health. http://www.who.int/phe

32. Zegmout M., Basraoui Y., Meziane M., Chahlaoui A., Demnati S., Chafi A., 2011. Pollution bactériologique de la zone côtière Saidia/Moulouya (région orientale du Maroc). Rev. Microbiol. Ind. San et Environn. 5 (2) 71-85. 